\title{
A produção científica sobre treinadores de futsal no Brasil
}

\author{
Scientific production about the futsal coaches in Brazil
}

\section{Producción científica en entrenadores de fútbol sala en Brasil}

(iD) Cesar Vieira Marques Filho

Universidade Estadual de Campinas, Campinas, São Paulo, Brasil.

cesarvmf@hotmail.com

iD Gustavo Sanchez Folhas

Universidade Estadual de Campinas, Limeira, São Paulo, Brasil.

gustavofolhas@gmail.com

iD (9) Larissa Rafaela Galatti

Universidade Estadual de Campinas, Limeira, São Paulo, Brasil.

lagalatti@hotmail.com

iD Wilton Carlos de Santana

Universidade Estadual de Londrina, Londrina, Paraná, Brasil.

wilton@pedagogiadofutsal.com.br

iD (9) Paulo Cesar Montagner

Universidade Estadual de Campinas, Campinas, São Paulo, Brasil. cesar.montagner@fef.unicamp.br

Resumo: Objetivo: compreender a produção científica sobre treinadores de Futsal no Brasil. Método: revisão de literatura a partir dos periódicos nacionais relacionados ao esporte. Resultados: pico de publicações entre 2012 e 2017; Sul e Sudeste como regiões com mais publicações; predomínio de artigos empíricos; equilíbrio entre metodologias quali-quanti-mistas; métodos de ensino-treino como principal temática complementar e equilíbrio entre pesquisas com Futsal de rendimento 
e participação. Conclusão: o estado da arte sobre o treinador de Futsal no Brasil possui um caráter recente, composto por um número ainda reduzido de obras. Esta revisão almeja facilitar o acesso a estes estudos e instigar outros autores a produzir pesquisas voltadas aos treinadores de Futsal.

Palavras-chave: Futsal. Treinador. Produção Científica.

Abstract: Objective: to understand the scientific production on the futsal coach in Brazil. Method: literature review from national journals related to the sport. Results: peak of publications between 2012 and 2017; The South and Southeast regions have the most publications; predominance of empirical articles; balance between quali-quanti-mixed methodologies; teaching-trainning methods as the main theme, and balance between performance and recreational research. Conclusion: the state of the art on the futsal coach in Brazil is recent, composed still by a reduced number of papers. This review aims to ease the access to these studies and encourage authors to produce research focused on futsal coaches.

Keywords: Futsal. Coach. Scientific Production.

Resumen: Objetivo: comprender la producción científica sobre entrenadores de fútbol sala en Brasil. Método: revisión de la literatura basada en revistas relacionadas con el deporte. Resultados: pico de publicaciones entre 2012 y 2017; Sur y Sudeste como regiones con más publicaciones; predominio de artículos empíricos; equilibrio entre metodologías cuali-cuanti-mezcladas; métodos de enseñanzaentrenamiento como tema principal y equilibrio entre la investigación sobre rendimiento y participación. Conclusión: el estado del arte en entrenadores de fútbol sala en Brasil tiene un carácter reciente, compuesto de pocas obras. Esta revisión tiene como objetivo facilitar el acceso a estos estudios y alentar a otros autores a producir investigaciones dirigidas a los entrenadores de fútbol sala.

Palabras-clave: Fútbol Sala. Entrenador. Producción Científica.

Submetido em: 23-07-2020

Aceito em: 29-09-2020 


\section{Introdução}

O esporte caracteriza-se por um fenômeno plural e social, praticado em diferentes contextos e com distintas finalidades. No Brasil, o Futsal é uma das modalidades mais populares, abarcando aproximadamente 12 milhões de praticantes, conforme o Ministério da Cidadania (2016). Sob a tutela da FIFA, a partir da década de 1990, o Futsal começa o seu período de profissionalização, passando a ser visto como produto de entretenimento e tornando-se objeto de estudo para áreas como a fisiologia, a pedagogia e a psicologia (SANTANA, 2004; RUFINO; DARIDO, 2011; MORALES JÚNIOR et al., 2017). Dentro desse contexto amplo, com inúmeros personagens envolvidos no âmbito do Futsal, neste estudo, aprofundamos o olhar acerca da figura do treinador, profissional que ganha destaque por possuir funções variadas, atuando como técnico esportivo, gestor, líder e educador (JONES, 2006; CÔTÉ; GILBERT, 2009), contendo um vínculo essencialmente pedagógico e social (MESQUITA et al., 2012).

O reconhecimento da importância do treinador gerou uma série de iniciativas voltadas para esse profissional no âmbito mundial. Houve a ampliação dos investimentos realizados em programas de formação, objetivando o aumento na qualidade da preparação desses profissionais nos contextos formal e não formal (LYLE, 2002; LYLE, 2007; WERTHNER; CURVER; TRUDEL, 2012; SHERIDAN, 2014; GALATTI; SANTOS; KORSAKAS, 2019), além do estabelecimento de padrões internacionais que possibilitem a sua atuação em diferentes países (ICCE, 2013). As discussões sobre o treinador, no contexto acadêmico e nas respectivas produções científicas, também se intensificaram nos últimos anos (DUFFY et al., 2011; MALCOM; PINHEIRO; PIMENTA, 2014; SHERIDAN, 2014).

No contexto brasileiro, o treinador também passou a ser tema de pesquisas acadêmicas. Entretanto, as produções científicas sobre os treinadores esportivos ainda são incipientes (NUNOMURA et al., 2011; RAMOS et al., 2011; MILISTETD et al., 2016). Para melhor 
A produção científica sobre treinadores de futsal no Brasil

Cesar Vieira Marques Filho • Gustavo Sanchez Folhas • Larissa Rafaela Galatti • Wilton Carlos de Santana $\cdot$ Paulo Cesar Montagner

compreensão deste cenário, Galatti et al. (2016) realizaram uma revisão de literatura sobre os treinadores esportivos, abarcando todas as modalidades, e encontraram 82 obras publicadas nos periódicos nacionais de 2009 a 2015. O Futsal foi a sexta modalidade com mais estudos acerca do treinador, com um total de 10 obras. Contudo, nesta revisão, não se adentrou na especificidade dos estudos com essa modalidade, sendo apresentado um panorama geral.

Um olhar mais específico a respeito da produção sobre o Futsal foi realizado por Caregnato et al.(2015), ao construírem um levantamento acerca das dissertações e teses dos programas de pós-graduação no âmbito nacional entre os anos de 1996 e 2012. De um total de 96 estudos, o termo "treinador" consta no título de uma obra e nos objetivos de outras três, de forma que não se tem uma discussão mais aprofundada sobre a temática do treinador em particular.

Embora as revisões supracitadas contribuam para o entendimento do estado da arte do treinador esportivo e do Futsal no Brasil, ainda persiste uma lacuna em relação a um olhar mais detaIhado e específico sobre estas duas temáticas em conjunto. Ainda, ao abranger um recorte temporal mais amplo, que englobe desde o início das publicações acerca do treinador de Futsal até os dias atuais (2019), temos a possibilidade de uma melhor compreensão deste contexto. Neste sentido, o estudo tem por objetivos mapear e discutir a produção científica sobre os treinadores de Futsal, a partir de artigos publicados nos periódicos nacionais que tem o esporte como seu escopo.

\section{Materiais e métodos}

A metodologia de revisão de literatura utilizada neste estudo foi o "estado da arte". Ela permite mapear e discutir a produção acadêmica em determinado campo de conhecimento, compreendendo os elementos priorizados na produção literária em deter- 
A produção científica sobre treinadores de futsal no Brasil

Cesar Vieira Marques Filho • Gustavo Sanchez Folhas • Larissa Rafaela Galatti • Wilton Carlos de Santana Paulo Cesar Montagner

minada época e contexto (FERREIRA, 2002; BARREIRA et. al., 2018). Com o intuito de discutir a produção científica brasileira, este estudo delimitou seu universo investigado a partir dos periódicos nacionais que publicam estudos sobre o esporte e possuem indexação pela Qualis Capes na área 21 (2013-2016), com base em Barreira et al. (2018). Consideramos todas as pesquisas publicadas até o final de 2019. Fizeram parte do escopo desta revisão tanto periódicos com frequência de publicações ativas, quanto aqueles que encerraram suas atividades mas mantêm seu acervo com possibilidade de acesso.

Inicialmente, utilizamos as palavras-chave "treinador" e "futsal" para realização da coleta dos artigos. Todavia, com a progressão nas leituras da temática, sentimos a necessidade de acrescentar os termos "técnico" e "professor". A tríade treinador-técnico-professor se faz necessária neste estudo, pois essas nomenclaturas são comumente utilizadas na cultura esportiva brasileira, principalmente no que tange ao Futsal e ao Futebol (BENITES; BARBIERI; SOUZA NETO, 2007) e são, portanto, imprescindíveis para a abrangência pretendida nesta revisão. Cabe ressaltar que não consideramos o termo "técnico" como ideal, pois apresenta um caráter redutivo da atuação do treinador como apenas um aplicador de técnicas (SCAGLIA, 2018). Contudo, entendemos que a utilização de "professor" se faz adequada partir do entendimento do viés pedagógico desta profissão, ou seja, o professor/treinador enquanto um agente que tem por responsabilidade mediar um processo de ensino-aprendizagem (PEREIRA; MESQUITA; GRAÇA, 2010; SCAGLIA 2018). Por fim, a utilização das palavras de forma mais neutra permitiu abrangê-las no masculino e feminino, bem como no singular e plural.

Como critério de inclusão para a coleta, consideramos todos os artigos em que constassem as palavras-chave. Na sequência, adotamos os seguintes critérios de exclusão: 1) Estudos que não fossem artigos originais ou revisões de literatura; 2) A partir da leitura dos resumos, artigos que não tivessem no treinador de Futsal a sua temática central; 3) A partir da leitura na íntegra, artigos 
A produção científica sobre treinadores de futsal no Brasil

Cesar Vieira Marques Filho • Gustavo Sanchez Folhas • Larissa Rafaela Galatti • Wilton Carlos de

Santana $\cdot$ Paulo Cesar Montagner

que não tivessem no treinador de Futsal a sua temática central; 4) Artigos que tratassem de mais alguma modalidade e os resultados não fossem apresentados separadamente para o treinador de Futsal.

Para a categorização dos resultados, utilizamos critérios apresentados em revisões de literatura com proximidades temáticas (RUFINO; DARIDO, 2011; GALATTI et al., 2016; BARREIRA et al., 2018; COSTA et al., 2019). Desta forma, os resultados foram agrupados em: 1) Ano de publicação; 2) Distribuição geográfica; 3) Composição metodológica; 4) Temática complementar e 5) Contextos do treinador. A categorização dos artigos foi realizada, conjuntamente, por dois autores. Outros três autores, com formação em nível de doutorado e autores de produções científicas sobre a temática deste estudo, realizaram a verificação da adequação deste processo. Os dados foram organizados através de uma planilha do Microsoft Office Excel e a apresentação dos resultados foi feita por meio de estatística descritiva, a partir de frequências relativas e absolutas.

\section{Resultados e discussão}

Foram encontrados 474 artigos a partir da utilização das palavras-chave. Após a aplicação dos critérios de exclusão, restaram 26 artigos, que compõem os resultados desta revisão de literatura. Todos estão listados ao final do estudo. O panorama geral vem apresentado no Quadro 1: 
A produção científica sobre treinadores de futsal no Brasil Cesar Vieira Marques Filho - Gustavo Sanchez Folhas • Larissa Rafaela Galatti • Wilton Carlos de Santana $\cdot$ Paulo Cesar Montagner

\section{Quadro 1 - Distribuição dos artigos que compõe o estudo}

\begin{tabular}{lcc}
\hline \multicolumn{1}{c}{ Título do periódico } & $\begin{array}{c}\text { Estrato Qualis } \\
(\mathbf{2 0 1 3 - 2 0 1 6 )}\end{array}$ & $\begin{array}{c}\text { Frequência de Artigos } \\
\text { Absoluta (Relativa) }\end{array}$ \\
\hline $\begin{array}{lcc}\text { Coleção Pesquisas em Educação Física } \\
\begin{array}{l}\text { Revista Mackenzie de Educação Física e Es- } \\
\text { porte }\end{array}\end{array}$ & B4 & $1(3,8 \%)$ \\
Pensar a Prática & B2 & $1(3,8 \%)$ \\
Motriz & B1 & $1(3,8 \%)$ \\
Revista Brasileira de Ciência e Movimento & B2 & $2(7,6 \%)$ \\
Conexões & B4 & $2(7,6 \%)$ \\
Movimento & A2 & $3(11,5 \%)$ \\
Revista Brasileira de Futsal e Futebol & B4 & $3(11,5 \%)$ \\
\hline TOTAL & & $\mathbf{2 6}(\mathbf{1 0 0 \% )}$ \\
\hline
\end{tabular}

O ano de publicação de cada artigo encontrado é apresentado na Figura 1. Não existem produções anteriores aos anos 2000 e há um crescimento acentuado no final da década seguinte. Este contexto é similar aos achados referentes às produções sobre o Futsal em dissertações e teses (CAREGNATO et al, 2015), aos treinadores esportivos sem discriminação por modalidade (GALATTI et al., 2016) e à pedagogia do esporte até o ano de 2010 (RUFINO; DARIDO, 2011), área que engloba as temáticas próximas ao presente estudo. 
A produção científica sobre treinadores de futsal no Brasil

Cesar Vieira Marques Filho • Gustavo Sanchez Folhas • Larissa Rafaela Galatti • Wilton Carlos de

Santana $\cdot$ Paulo Cesar Montagner

Figura 1 - Ano de publicação

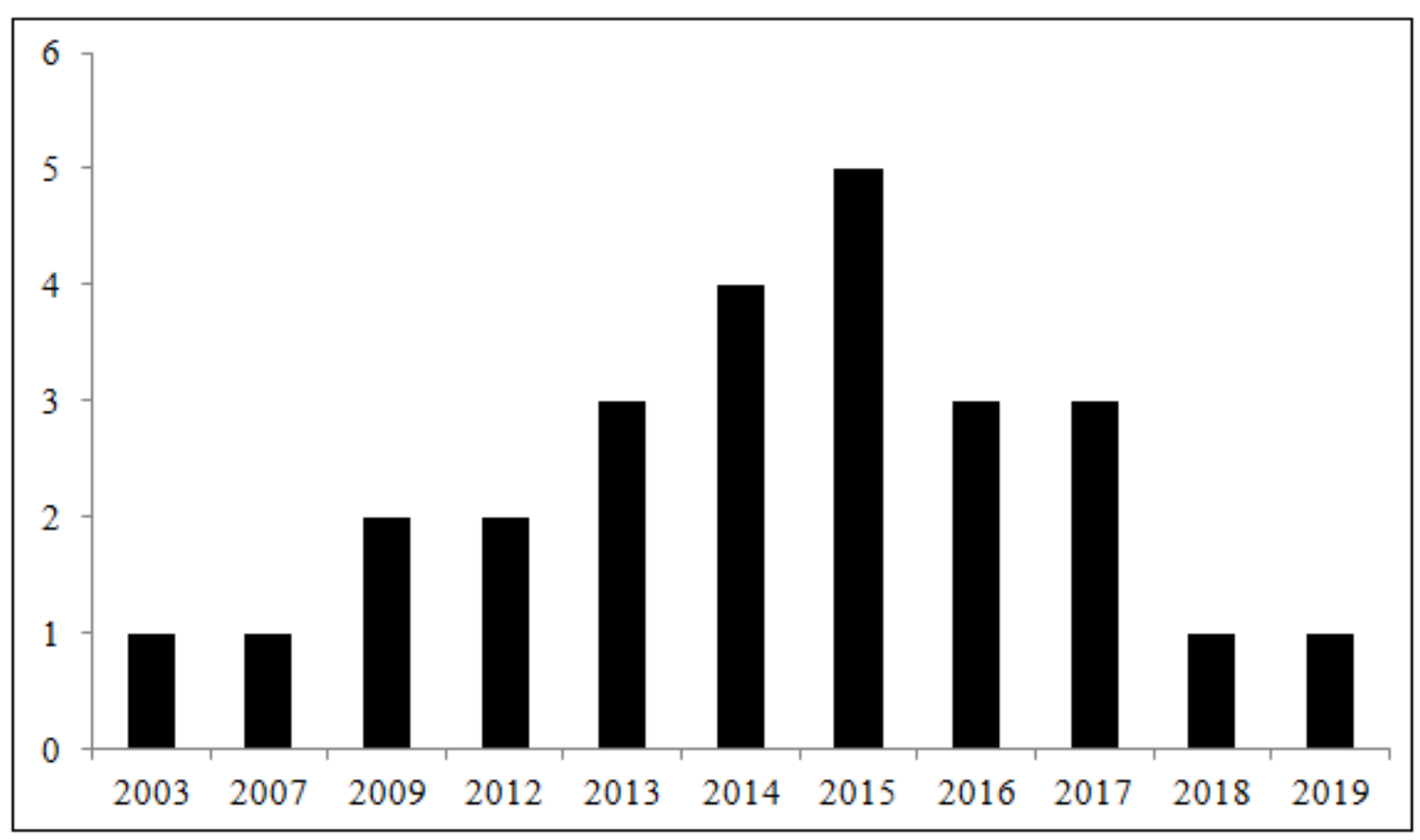

Um dos fatores que justifica este panorama está no aporte financeiro do governo brasileiro para o desenvolvimento de programas de pós-graduação em Educação Física e Esportes, impulsionando a produção acadêmica na área (GENTIL; BRITO NETO, 2015; CAREGNATO et al., 2015; GALATTI et al., 2016). Outro elemento que ganha destaque é o início das publicações da Revista Brasileira de Futsal e Futebol (RBFF) no ano de 2009, periódico responsável por metade das produções encontradas nesta revisão. O mesmo impacto é percebido em Barreira et al. (2018), no qual as autoras apontam que a RBFF publica estudos de professores, estudantes e profissionais que atuam no Futebol e/ou Futsal, da iniciação ao alto rendimento, abarcando questões pedagógicas, sociais e de treinamento.

A realização de megaeventos esportivos também pode contribuir para elevar o número de pesquisas na área no contexto nacional. Esta relação é indicada por alguns autores (ALMEIDA; MEZZADRI; MARCHI JÚNIOR, 2009; GALATTI et al., 2016, AZEVEDO et al., 2017) e corrobora os achados deste estudo. Os Jogos Pan- 
A produção científica sobre treinadores de futsal no Brasil

Cesar Vieira Marques Filho • Gustavo Sanchez Folhas • Larissa Rafaela Galatti • Wilton Carlos de

Santana $\cdot$ Paulo Cesar Montagner

Americanos foram sediados no Brasil em 2007 (única edição na qual o Futsal esteve presente) e em 2008 foi realizada a Copa do Mundo de Futsal. Na mesma medida em que esses megaeventos podem ter alavancado as produções nos anos seguintes, um efeito antagônico também pode ter ocorrido com a Copa do Mundo de Futebol, sediada em 2014, e os Jogos Olímpicos, em 2016. As pesquisas com treinadores podem ter sido voltadas aos esportes pertencentes a esses megaeventos, justificando o declínio nas pesquisas com Futsal nos anos seguintes (2018 e 2019).

A Figura 2 ilustra a produção dos artigos a partir de um ponto de vista geográfico. Como critério, utilizamos a instituição de filiação dos primeiros autores de cada estudo e seu respectivo local de pertencimento. Ao todo, encontramos artigos provenientes de oito estados, englobando três diferentes regiões.

Figura 2 - Distribuição geográfica dos artigos

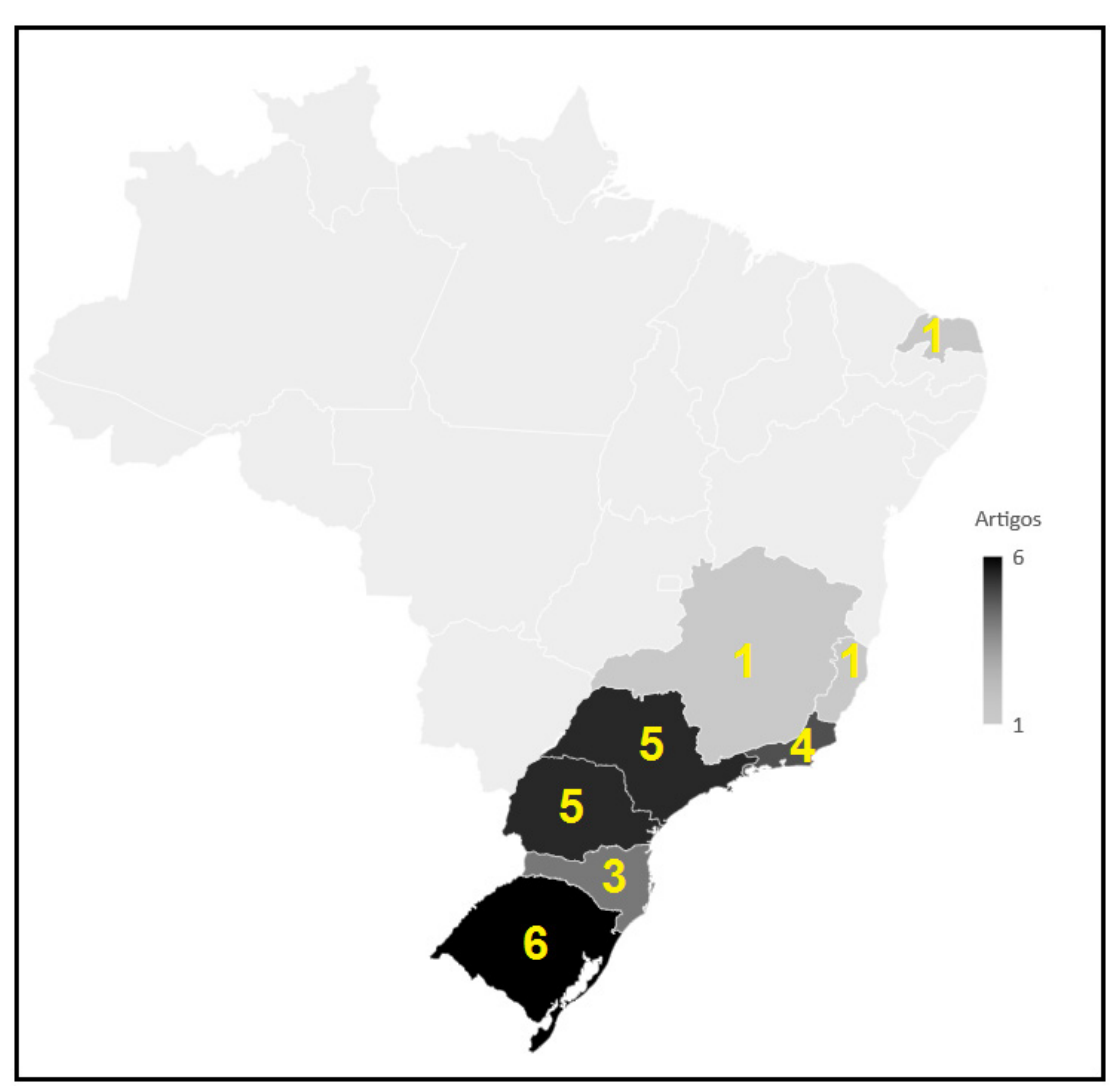


A produção científica sobre treinadores de futsal no Brasil

Cesar Vieira Marques Filho • Gustavo Sanchez Folhas • Larissa Rafaela Galatti • Wilton Carlos de Santana $\cdot$ Paulo Cesar Montagner

As regiões Sul e Sudeste abrangem quase a totalidade dos artigos encontrados, com exceção de um estudo proveniente do Rio Grande do Norte, no Nordeste. Ao analisarem a produção científica nacional como um todo, Sidone, Haddad e Mena-Chalco (2016) apontam um panorama semelhante, com essas regiões englobando $91,3 \%$ dessas produções. Todavia, os autores apresentam prevalência do Sudeste (54,3\%), seguido de Sul (22,1\%) e Nordeste $(14,9 \%)$. Na especificidade dos artigos sobre treinadores de Futsal, encontramos o Sul com $56 \%$, Sudeste com $40 \%$ e Nordeste com $4 \%$. A forte relação da região Sul com o Futsal justifica o presente cenário. Este contexto pode ser exemplificado ao revisarmos as equipes participantes de todas as edições da principal competição de Futsal do país, a Liga Nacional de Futsal. Segundo dados do site oficial ${ }^{1}$, o Sul abarca $70 \%$ das equipes, seguido do Sudeste com $27 \%$ e apenas $1 \%$ dividido entre as demais regiões. Dentre os estados, o Rio Grande do Sul aparece como o detentor do maior número de artigos. Segundo Vicari (2015), o Futsal possui um grande número de praticantes e elevada visibilidade neste estado e, conforme Brito e Bruscato (1995), esse contexto é proveniente da disseminação de sua modalidade de origem, o futebol de salão, na década de 1940.

O Quadro 2 apresenta a abordagem metodológica dos artigos. Os dados foram agrupados por seu caráter teórico e empírico e segundo as metodologias empregadas, a partir dos critérios estipulados por Creswell (2003), sendo classificados como qualitativos, quantitativos e mistos. Ainda, foram apresentadas as ferramentas de coletas de dados utilizadas em cada estudo.

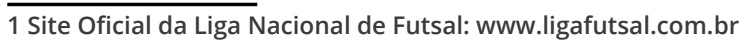


A produção científica sobre treinadores de futsal no Brasil

Cesar Vieira Marques Filho - Gustavo Sanchez Folhas • Larissa Rafaela Galatti • Wilton Carlos de

Santana $\cdot$ Paulo Cesar Montagner

Quadro 2 - Composição Metodológica

\begin{tabular}{ccc}
\hline & \multicolumn{2}{c}{ Artigos Teóricos } \\
\hline $\begin{array}{c}\text { Metodologia } \\
\text { Qualitativa }\end{array}$ & $\begin{array}{c}\mathbf{N}^{\circ} \text { de artigos } \\
4(15,3 \%)\end{array}$ & $\begin{array}{c}\text { Ferramenta de coleta } \\
\text { Ensaio (3), Revisão bibliográfica (1) }\end{array}$ \\
\hline Metodologia & $\mathbf{N}^{\circ}$ de artigos & Artigos Empíricos \\
Qualitativa & $8(30,7 \%)$ & Ferramenta de coleta \\
Quantitativa & $7(26,9 \%)$ & Entrevta (4), Relato de experiência (3), Observação (1) \\
Mista & $7(26,9 \%)$ & Questionário (7) \\
& & \\
\hline
\end{tabular}

Apenas $15,3 \%$ dos artigos são de cunho teórico, enquanto $84,7 \%$ possuem uma abordagem empírica. Conforme Gilbert e Trudel (2004), com o aumento da importância dada ao treinador esportivo, surgem mais estudos que, para além da discussão no campo teórico, buscam encontrar evidências empíricas para sustentar o marco teórico a respeito do tema. Inicialmente, as pesquisas sobre o treinador esportivo foram pautadas em uma perspectiva majoritariamente quantitativa, proveniente de uma influência positivista na concepção de ciência dos pesquisadores (SILVERMAN; SKONIE, 1997; CULVER; GILBERT; SPARKES, 2012). Com o decorrer dos anos, os estudos qualitativos passaram a ocupar mais espaço (GILBERT; TRUDEL, 2004), possibilitando um maior aprofundamento no universo de significados, motivos, aspirações, crenças, valores e atitudes, o que corresponde a buscar mais profundidade nas relações e nos fenômenos, que não podem ser reduzidos à mera operacionalização de variáveis (MINAYO, 2011). Devido ao caráter recente das publicações acerca do treinador de Futsal no Brasil, percebemos que existe um equilíbrio na tríade quanti-quali-misto, característica positiva, na medida em que proporciona o estudo do fenômeno a partir de diferentes perspectivas.

As ferramentas de coleta de dados demonstram a preocupação em identificar a perspectiva e opiniões dos treinadores sobre determinados assuntos, na medida em que se destacam os questionários e entrevistas. Gilbert e Trudel (2004) salientam a impor- 
A produção científica sobre treinadores de futsal no Brasil

Cesar Vieira Marques Filho - Gustavo Sanchez Folhas • Larissa Rafaela Galatti • Wilton Carlos de

Santana $\cdot$ Paulo Cesar Montagner

tância desse cenário, considerando imprescindível que os estudos na área transcendam a perspectiva dos pesquisadores e consigam acessar as ideias e pensamentos dos treinadores. Entretanto, é importante a utilização de diferentes ferramentas de coleta de dados quando se estuda o treinador esportivo (POTRAC et al., 2000), panorama não encontrado nas pesquisas com o Futsal brasileiro. Nelas, apenas cinco artigos mesclam diferentes ferramentas, sendo que, nos cinco casos, a observação foi utilizada como complemento das informações obtidas por questionários ou entrevistas.

O Quadro 3 apresenta os temas complementares de cada artigo em relação ao treinador de Futsal. A partir da leitura crítica de cada estudo, foram elaboradas nove categorias de aproximação temática.

Quadro 3 - Temática Complementar

\begin{tabular}{lc}
\hline \multicolumn{1}{c}{ Temática } & Número de artigos \\
\hline Metodologia de ensino-treino & $6(24 \%)$ \\
Conhecimentos e competências & $3(12 \%)$ \\
Perfil de treinadores & $3(12 \%)$ \\
Motivação à prática & $3(12 \%)$ \\
Planejamento e sistematização & $3(12 \%)$ \\
Liderança & $2(8 \%)$ \\
Estratégia e tática & $2(8 \%)$ \\
Valores sociais & $2(8 \%)$ \\
Competição & $1(4 \%)$ \\
\hline
\end{tabular}

Percebemos a ênfase nos estudos que abordam o aspecto metodológico do ensino-treino. No Brasil, este contexto de discussão ganha destaque, principalmente a partir da década de 1990 (PAES, 1996; GRECO, 1998; FREIRE, 1998). Todavia, os debates e impasses mantiveram-se presentes e, desde então, ano após ano, diferentes autores apontam essa temática como uma discussão ampla e atual no contexto brasileiro (GAYA, 2000; COSTA; NASCIMENTO, 2004; PÉREZ MORALES; GRECO, 2007; COUTINHO; SILVA, 2009; ROMÃO; BARBOSA; MOREIRA, 2017), a ponto de predominar for- 
A produção científica sobre treinadores de futsal no Brasil

Cesar Vieira Marques Filho • Gustavo Sanchez Folhas • Larissa Rafaela Galatti • Wilton Carlos de Santana $\cdot$ Paulo Cesar Montagner

temente nos estudos em Pedagogia do Esporte no país entre 2010 e 2015 (COSTA et al., 2019). Desta forma, esse contexto direciona os aspectos metodológicos como uma das principais preocupações quanto à atuação do treinador nos artigos encontrados nesta revisão.

Além dos aspectos metodológicos, as sete temáticas seguintes (totalizando $96 \%$ dos artigos) correspondem às categorias "thinking" e "behavior", apresentadas por Galatti et al. (2016), sendo as principais abordadas por artigos que estudam treinadores esportivos. Elas dizem respeito ao que os treinadores pensam ou sentem e o que fazem ou deveriam fazer, respectivamente. Todavia, outras categorias de destaque, como desenvolvimento de carreira, avaliação e discussão de gênero (GALATTI et al., 2016), não foram encontradas nos estudos com treinadores de Futsal no Brasil.

O Quadro 4 diz respeito aos contextos de atuação do treinador abordados em cada artigo. Para este item, foram retirados quatro estudos que não apresentavam um contexto específico. $O$ agrupamento dos resultados foi realizado a partir do ambiente de atuação do treinador, bem como sua finalidade. A partir das classificações de Lyle (2002) e Cótê e Gilbert (2009), pesquisas que abordaram um treinador com viés prioritariamente competitivo foram classificadas como "Futsal de rendimento", enquanto as que se referem ao treinador que tem por finalidade aspectos como diversão e ludicidade, com menor componente competitivo, foram classificadas como "Futsal de participação". Desta forma, uma equipe escolar que busca resultados competitivos e performance esportiva pode ser classificada como Futsal de rendimento, por exemplo. 
A produção científica sobre treinadores de futsal no Brasil

Cesar Vieira Marques Filho - Gustavo Sanchez Folhas • Larissa Rafaela Galatti • Wilton Carlos de

Santana $\cdot$ Paulo Cesar Montagner

Quadro 4 - Contextos do Treinador

\begin{tabular}{cccc}
\hline Contexto & Futsal de rendimento & $\begin{array}{l}\text { Futsal de participa- } \\
\text { ção }\end{array}$ & Total \\
\hline Categorias de base & $4(19 \%)$ & $2(9 \%)$ & $6(28 \%)$ \\
Escola & $2(9 \%)$ & $5(24 \%)$ & $7(33 \%)$ \\
Escolinhas de iniciação & 0 & $3(14 \%)$ & $3(14 \%)$ \\
Adulto & $4(19 \%)$ & $1(5 \%)$ & $5(24 \%)$ \\
\hline Total & $\mathbf{1 0 ( 4 8 \% )}$ & $\mathbf{1 1 ( 5 2 \% )}$ & $\mathbf{2 1 ( 1 0 0 \% )}$ \\
\hline
\end{tabular}

Existe um equilíbrio entre os estudos que abordam treinadores que têm por finalidade o Futsal de participação e o de rendimento. Todavia, apenas quatro pesquisas abordam o Futsal adulto de rendimento, apesar da existência de maior visibilidade e investimentos para esse segmento. Williams e Kendall (2007) apontam a dificuldade em unir interesses de pesquisadores e treinadores em contextos profissionais ou de alto rendimento. Os mesmos autores revelam que as pesquisas científicas na área levam muito tempo para responder às perguntas dos treinadores. De maneira semelhante, em revisão realizada por Gilbert e Trudel (2004), encontra-se o distanciamento entre o esporte de elite e as produções científicas acerca dos treinadores esportivos. A linguagem científica, a dificuldade de acesso aos periódicos e artigos e a desvalorização do conhecimento científico são apontados como os principais fatores que impedem a aproximação entre pesquisadores e treinadores (GILBERT; TRUDELL, 2004; WILLIAMS; KENDALL, 2007). Consequentemente, os artigos científicos parecem não estar entre as principais fontes de conhecimento dos treinadores que tendem a optar, principalmente, pela troca entre pares e colegas e presença em clínicas e workshops (WILLIAMS; KENDALL, 2007).

Quanto à finalidade de participação para o Futsal adulto, a ocorrência é ainda menor, com apenas um artigo publicado. Este cenário pode estar atrelado ao negligenciamento do treinador neste segmento, pois não está associado nem ao desenvolvimento de crianças e jovens, nem à finalidade prioritariamente competitiva do esporte de rendimento (LYLE, 2002). Conforme Cótê e Gilbert 
A produção científica sobre treinadores de futsal no Brasil

Cesar Vieira Marques Filho • Gustavo Sanchez Folhas • Larissa Rafaela Galatti • Wilton Carlos de Santana $\cdot$ Paulo Cesar Montagner

(2009), esse treinador tem como responsabilidade proporcionar o desenvolvimento de interações sociais, competitividade sadia e condicionamento físico voltados ao bem-estar físico e social. Neste contexto, o treinador trabalha a partir de metas de curto prazo, estabelece desafios pessoais ao invés de comparações entre os praticantes, portanto, seus resultados devem ser ponderados na esfera da saúde e satisfação dos atletas como participantes (LYLE, 2002).

Dentre os diferentes contextos, ganha destaque o de treinadores de crianças e jovens, principalmente no ambiente escolar (33\%). Os achados vão ao encontro do panorama apontado por Sá (2009), no qual o Futsal é o esporte mais praticado por crianças e jovens no Brasil, sobretudo nas escolas. Conforme Voser (2003), o Futsal pode proporcionar inúmeros benefícios a esses estudantes, desde os aspectos físicos até os sociais, como a melhora da qualidade de vida (BISCAIA, 2020) e autoconfiança (FERNANDES et al., 2013), desde que sua prática seja conduzida de forma positiva, evitando a competitividade exacerbada e a exclusão dos menos habilidosos. Neste sentido, destacamos a importância do treinador na construção de um ambiente de competição com viés pedagógico, proporcionando um contexto educativo que contribua positivamente para a formação de seus participantes (MONTAGER; SCAGLIA, 2013).

\section{Considerações finais}

Por meio desta revisão, pudemos compreender que o estado da arte sobre o treinador de Futsal no Brasil possui um caráter recente, bem como é composto por um número ainda reduzido de obras. A ciência brasileira tem potencial para oferecer mais contribuições sobre a temática, que engloba uma função tão importante quanto a do treinador em uma modalidade tão disseminada no país quanto o Futsal. 
A produção científica sobre treinadores de futsal no Brasil

Cesar Vieira Marques Filho • Gustavo Sanchez Folhas • Larissa Rafaela Galatti • Wilton Carlos de Santana $\cdot$ Paulo Cesar Montagner

A temática mais estudada sobre o treinador de Futsal está relacionada aos métodos de ensino-treino, refletindo em uma das principais discussões sobre contexto esportivo brasileiro no âmbito das ciências do esporte. É interessante perceber que, na maior parte dos estudos, os pesquisadores têm buscado compreender a perspectiva dos treinadores sobre determinados assuntos, o que pode sinalizar um importante processo de construção conjunta entre os profissionais da ciência e os inseridos no campo prático, e não, uma tentativa de imposição de verdades absolutas de um sobre o outro.

Como implicações práticas, através do mapeamento de todos os artigos que abordam o treinador de Futsal, esta revisão almeja facilitar o acesso dos sujeitos interessados na temática a esses estudos, disponibilizando-os em uma lista anexa. Ainda, esperamos que a apresentação deste panorama possa instigar outros autores a produzir pesquisas voltadas aos treinadores de Futsal.

\section{Referências}

ALMEIDA, B. S.; MEZZADRI, F. M.; MARCHI JÚNIOR, W.

Considerações sociais e simbólicas sobre sedes de megaeventos esportivos. Motrivivência, Florianópolis, v. 21, n. 32/33, p. 178192, jun/dez. 2009.

AZEVEDO, M. F. et al. Formação escolar e formação esportiva: caminhos apresentados pela produção acadêmica. Movimento, Porto Alegre, v. 23, n. 1, p. 185-200, jan/mar. 2017.

BARREIRA, J. et al. Produção acadêmica em futebol e futsal feminino: estado da arte dos artigos científicos nacionais na área da Educação Física. Movimento, Porto Alegre, v. 24, n. 2, p. 607-618, abr/jun. 2018.

BENITES, L. C.; BARBIERI, F. A.; SOUZA NETO, S. O futebol: questões e reflexões a respeito dessa "profissão". Pensar a Prática, Goiânia, v. 10, n. 1, p. 51-67, jan/jun. 2007. 
A produção científica sobre treinadores de futsal no Brasil

Cesar Vieira Marques Filho - Gustavo Sanchez Folhas • Larissa Rafaela Galatti • Wilton Carlos de Santana $\cdot$ Paulo Cesar Montagner

BISCAIA, R. R. Associação do autoconceito, autoeficácia e qualidade de vida entre escolares de 15 e 17 anos praticantes e não praticantes de futsal em Curitiba. 2020. 109f. Dissertação (Mestrado em Educação) - Setor de Educação, Universidade Federal do Paraná, Curitiba, 2020.

BRITO, P.; BRUSCATO, R. Futsal Gaúcho. Porto Alegre: Independente, 1995.

CAREGNATO, A. P. et al. Produção científica sobre futsal: análise de dissertações e teses publicadas no portal da Capes entre 1996-2012. Motrivivência, Florianópolis, v. 27, n. 46, p. 15-34, dez. 2015.

COSTA, L. C. A.; NASCIMENTO, J. V. O ensino da técnica e da tática: novas abordagens metodológicas. Rev. da Educação Física/ UEM, Maringá, v. 15, n. 2, p. 49-56, jul/dez. 2004.

COSTA, R. R. et al. Pedagogia do esporte: publicações em periódicos científicos brasileiros de 2010 a 2015. Conexões, Campinas, v. 17, e019008, p. 1-18, 2019.

CÔTÉ, J.; GILBERT, W. D. An Integrative Definition of Coaching Effectiveness and Expertise, International Journal of Sports Science \& Coaching, v. 4, n. 3, p. 307-323, 2009.

COUTINHO, N. F.; SILVA, S. S. Conhecimento e Aplicação de Métodos de Ensino para os Jogos Esportivos Coletivos na Formação Profissional em Educação Física. Movimento, Porto Alegre, v. 15, n. 1, p. 117-144, jan/mar. 2009.

CRESWELL, J. W. Research design: Qualitative, quantitative, and mixed method approaches. 2 ed. Thousand Oaks, CA: Sage, 2003. CULVER, D.; GILBERT, W.; SPARKES, A. Qualitative research in sport psychology journals: The next decade 2000-2009 and beyond. The Sport Psychologist, v. 26, n. 2, p. 261-281, 2012.

DUFFY, P. et al. Sport coaching as a 'profession'. International Journal of Coaching Science, v. 5, n. 2, p. 93-123, 2011.

FERREIRA, N. S. A. As pesquisas denominadas estado da arte. Educação \& Sociedade, v. 23, n. 79, p. 257- 272, ago. 2002. 
A produção científica sobre treinadores de futsal no Brasil

Cesar Vieira Marques Filho - Gustavo Sanchez Folhas • Larissa Rafaela Galatti • Wilton Carlos de Santana $\cdot$ Paulo Cesar Montagner

FERNANDES, M. G. et al. Factors influencing competitive anxiety in Brazilian athletes. Revista Brasileira de Cineantropometria \& Desempenho Humano, Florianópolis, v. 15, n. 6, p. 705-714, 2013.

FREIRE, J. B. Pedagogia do Futebol. Rio de Janeiro: Ney Pereira, 1998.

GALATTI, L. R. et al. Coaching in Brazil sport coaching as a profession in Brazil: an analysis of the coaching literature in Brazil from 2000-2015. International Sport Coaching Journal, v. 3, n. 3, p. 316-331, 2016.

GALATTI, L. R.; SANTOS, Y. Y. S.; KORSAKAS, P. A Coach

Developers' Narrative on Scaffolding a Learner-Centred Coaching Course in Brazil. International Sport Coaching Journal, v. 6, n. 3, p. 339-348, 2019.

GAYA, A. C. Sobre o esporte para crianças e jovens. Movimento, Porto Alegre, v.7, n.13, p.1-14, jul/dez. 2000.

GENTIL, R. N.; BRITO NETO, A. C. A expansão da pós-graduação em educação física no Brasil. XIX Congresso Brasileiro de Ciências do Esporte - VI Congresso Internacional de Ciências do Esporte, Vitória/ES, 2015.

GILBERT, W.; TRUDEL, P. Analysis of coaching science research published from 1970-2001. Research Quarterly for Exercise and Sport, v. 75, n. 4, p. 388-399, 2004.

GRECO, P. J. Iniciação esportiva universal. $2^{a}$ ed. Belo Horizonte: Ed. UFMG, 1998.

ICCE. International Council For Coaching Excellence. International Sport Coaching Framework Version 1.2. Champaign: Human Kinetics, 2013.

JONES, R. The Sports Coach as Educator: Re-conceptualising Sports Coaching. London: Routledge, 2006.

LYLE, J., Sports Coaching Concepts: A Framework for Coaches' Behaviour. Londres: Routledge, 2002. 
A produção científica sobre treinadores de futsal no Brasil

Cesar Vieira Marques Filho • Gustavo Sanchez Folhas • Larissa Rafaela Galatti • Wilton Carlos de Santana $\cdot$ Paulo Cesar Montagner

$L Y L E$, J. A review of the research evidence for the impact of coach education. International Journal of Coaching Science, v. 1, n. 1, p. 19-36, 2007.

MALCOM, D.; PINHEIRO, C.; PIMENTA, N. Could and should sport coaching become a profession? Some sociological reflections. International Sport Coaching Journal, v. 1, n. 1, p. 42-45, 2014. MESQUITA, l. et al. Nova Abordagem na Formação de Treinadores: O que mudou e Porquê? In: NASCIMENTO, J.; FARIAS, G. (eds.). Construção da Identidade Profissional em Educação Física: da Formação à Intervenção. Florianópolis: Coleção Movimento, 2012, p. 41-60.

MILISTETD, M. et al. Coaches' Development in Brazil: Structure of Sports Organizational Programmes. Sport Coaching Review, v. 5, n. 2, p. 138-152, 2016.

MINAYO, M. C. S. (Org.). Pesquisa Social: Teoria, Método e Criatividade. Petrópolis: Vozes, 2001.

MINISTÉRIO DA CIDADANIA. Secretaria Especial do Esporte. Notícias. Disponível em: http://arquivo.esporte.gov.br/index.php/ ver-todos/212-noticias/noticias-snfut. Acesso em: 22 nov. 2019

MONTAGNER, P. C.; SCAGLIA, A. Pedagogia da Competição:

Teoria e Proposta de Sistematização nas Escolas de Esportes. In: REVERDITO, R.; SCAGLIA, J. (Orgs.). Pedagogia do Esporte: Aspectos Conceituais da Competição e Estudos Aplicados. São Paulo: Phorte Editora, 2013, p. 193-216.

MORALES JÚNIOR, V. R. et al. The relative age effect on Brazilian elite futsal: Men and women scenarios. Motriz, Rio Claro, v. 23, n. 3, 2017.

NUNOMURA, M. et al. Ginástica Artística Competitiva e a Filosofia dos Técnicos. Motriz, Rio Claro, v. 18, n. 4, p. 678-689, out/dez. 2011.

PAES, R. R. Esporte Educacional: iniciação esportiva. In: INDESP Ministério dos Esportes. (Org.). Esporte Educacional: uma proposta renovada. 1 ed. Brasília: INDESP, 1996, p. 84-86. 
A produção científica sobre treinadores de futsal no Brasil

Cesar Vieira Marques Filho - Gustavo Sanchez Folhas • Larissa Rafaela Galatti • Wilton Carlos de Santana $\cdot$ Paulo Cesar Montagner

PEREIRA, F. R. M.; MESQUITA, I. M. R.; GRAÇA, A. B. S. A investigação sobre a eficácia pedagógica no ensino do desporto. Revista da Educação Física/UEM, Maringá, v. 21, n. 1, p. 147-160, jan/ mar. 2010.

PÉREZ MORALES, J. C.; GRECO, P. J. A influência de diferentes metodologias de ensino-aprendizagem-treinamento no basquetebol sobre o nível de conhecimento tático processual. Rev. Bras. Educ. Fís. Esp., São Paulo, v. 21, n. 4, p. 291-99, out/dez. 2007 POTRAC, P. et al. Toward an holistic understanding of the coaching process. Quest, v. 52, n. 2, p. 186-199, 2000.

RAMOS, V. et al. Aprendizagem Profissional: As Representações de Treinadores Desportivos de Jovens: Quatro Estudos de Caso. Motriz, Rio Claro, v. 17, n. 2, p. 280-291, abr./jun. 2011. ROMÃO, E. J. R.; BARBOSA, P. V. S.; MOREIRA, M. C. Metodologias de ensino para jogos esportivos coletivos na educação física escolar. Revista de Iniciação Científica da Universidade Vale do Rio Verde, Três Corações, v. 7, n. 1, p. 80-96, 2017.

RUFINO, L. G. B.; DARIDO, S. C. A produção científica em pedagogia do esporte: análise de alguns periódicos nacionais. Conexões, Campinas, v. 9, n. 2, p. 110-132, mai/ago. 2011.

\section{SÁ, T. A. A motivação de crianças entre 11 e 14 anos para} a prática do Futsal. 2009. 48f. Trabalho Conclusão de Curso (Licenciatura em Educação Física) - Faculdade de Educação Física, Universidade Federal do Rio Grande do Sul, Porto Alegre, 2009.

SANTANA, W. C. Futsal: apontamentos pedagógicos na iniciação e na especialização. 1 ed. Campinas: Autores Associados, 2004.

SCAGLIA, A. Treinador sim, técnico não. Universidade do Futebol, 2018. Disponível em: https://universidadedofutebol.com. br/treinador-sim-tecnico-nao/. Acesso em: 15 jan. 2020.

SHERIDAN, M. Could and Should Sport Coaching Become a Profession? Some Sociological Reflections: a comentary International Sport Coaching Journal, v.1, p. 46-49, 2014. 
A produção científica sobre treinadores de futsal no Brasil

Cesar Vieira Marques Filho • Gustavo Sanchez Folhas • Larissa Rafaela Galatti • Wilton Carlos de Santana $\cdot$ Paulo Cesar Montagner

SIDONE, O. J. G.; HADDAD, E. A.; MENA-CHALCO, J. P. A ciência nas regiões brasileiras: evolução da produção e das redes de colaboração científica. Transinformação, Campinas, v. 28, n.1, p. 15-31, jan/abr. 2016.

SILVERMAN, S.; SKONIE, R. Research on teaching in physical education: An analysis of published research. Journal of Teaching in Physical Education, v. 16, n. 3, p. 300-311, 1997.

VICARI, P. R. A transição do futebol de salão para o futsal: um percurso histórico no Rio Grande do Sul. 2015. 105f. Dissertação (Mestrado em Ciências do Movimento) - Escola de Educação Física, Universidade Federal do Rio Grande do Sul, Porto alegre, 2015.

VOSER, R. Futsal: princípios técnicos e táticos. Canos: ULBRA, 2003.

WERTHNER, P.; CULVER, D.; TRUDEL, P. An Examination of a Large Scale Coach Education Program From a Constructivist Perspective. In: SCHINKE, R. (ed.). Sport Psychology Insights. Londres: New Science Publishers Incp., 2012, p. 337-354.

WILLIAMS, J. S.; KENDALL, L. Perceptions of elite coaches and sports scientists of the research needs for elite coaching practice. Jounal of Sports Sciences, v. 25, n. 14, p. 1577-1586, 2007.

\section{Artigos que compõem a revisão}

1. ALVES, A.; NAVARRO, A.; A utilização da metodologia para o ensino-aprendizagem nas escolinhas de futsal para crianças com faixa etária de 6 á 10 anos. Revista Brasileira de Futsal e Futebol, Edição Especial: Pedagogia do Esporte, São Paulo, v. 7, n. 27, p. 474-494, jan/dez. 2015.

2. ALVES, M.; KVIATKOVSKI, B. Perfil dos professores/técnicos das equipes de futsal masculino da categoria "a" dos 
jogos estudantis da primavera: um comparativo dos anos de 2013 com o ano de 2015. Revista Brasileira de Futsal e Futebol, Edição Especial: Pedagogia do Esporte, São Paulo, v. 9, n. 35, p. 392-398, jan./dez. 2017.

3. ASSAD, D. et al. O valor do futsal como um meio de desenvolvimento moral de adolescentes com dificuldade de relacionamento social. Revista Brasileira de Futsal e Futebol, São Paulo, v. 5, n. 16, p. 146-154, mai/ago. 2013.

4. BANDEIRA, C.; ROdRIGUeS, H.; NAVARRO, A. Perfil dos técnicos de futsal feminino participantes dos jogos abertos do interior em 2008 no estado de São Paulo. Revista Brasileira de Futsal e Futebol, São Paulo, v. 1, n. 3, p. 193203, set/dez. 2009.

5. BetTeGA, O. B.; PRESTES, M. F.; LOPES, C.; GALATTI, L. Pedagogia do esporte: o jogo como balizador na iniciação ao futsal. Pensar a Prática, Goiânia, v. 18, n. 2, p. 487-501, abr./jun. 2015.

6. CAVALCANTI, L. Fatores que motivam alunos, professores e gestores na prática e desenvolvimento do futsal escolar. Revista Brasileira de Futsal e Futebol, Edição Especial: Pedagogia do Esporte, São Paulo, v. 5, n. 18, p. 284-290, jan/ dez. 2013.

7. DAOLIO, R.; MARQUES, R. Relato de uma experiência com o ensino de futsal para crianças de 9 a 12. anos. Motriz, Rio Claro, v.9, n.3, p. 169-174, set./dez. 2003.

8. JORAND, M. et al. A violência em categorias de base do futsal no Rio de Janeiro: um mergulho no universo dos pais e treinadores. Movimento, Porto Alegre, v. 25, n. 1, jan/dez. 2019.

9. KLEIN, R.; Métodos de ensino para o futsal escolar. Conexões, Campinas, v. 12, n. 4, p. 91-96, out/dez. 2014.

10.MARCON, M.; SAAD, M.; Estilo de tomada de decisão dos treinadores de equipes de futsal e futebol nas categorias de 
A produção científica sobre treinadores de futsal no Brasil

Cesar Vieira Marques Filho - Gustavo Sanchez Folhas • Larissa Rafaela Galatti • Wilton Carlos de Santana $\cdot$ Paulo Cesar Montagner

base. Revista Brasileira de Futsal e Futebol, São Paulo, v. 5, n. 17, p. 205-212, set/dez. 2013.

11.MARQUES, R. Entrevista com o ex-técnico da seleção Brasileira masculina de futsal, campeão mundial da modalidade em 2008 e atual técnico do S. Corinthians Paulista, Paulo César de Oliveira. Conexões, Campinas, v. 7, n. 3, p. 114-120, set/dez. 2009.

12. NASCIMENTO JUNIOR J. R. A; VIEIRA, L. F. Liderança do técnico e coesão de grupo:um estudo com equipes profissionais de futsal. Rev. Bras. Cien. Mov., Taguatinga, v. 20, n. 2, p. 84-90, set/dez. 2012.

13. NOVAES, R; RIGON, T; DANTAS, T. Modelo do jogo de futsal e subsídios para o ensino. Movimento, Porto Alegre, v. 20, n. 3, p. 1039-1060, jul./set. 2014.

14. OlIVETE, B. et al. Pressupostos teóricos que orientam o trabalho prático dos treinadores de escolas de iniciação de futsal. Revista Brasileira de Futsal e Futebol, Edição Especial: Pedagogia do Esporte, São Paulo, v. 7, n. 27, p.467473, jan/dez. 2015.

15. PORTUGAL, C.; NAZÁRIO, M. O estado do conhecimento sobre os saberes na formação do treinador de futebol e futsal. Revista Brasileira de Futsal e Futebol, São Paulo, v. 9, n. 34, p. 298-307, set/dez. 2017.

16.SAAD, M. et al. Impacto das metodologias empregadas pelos treinadores no desenvolvimento técnico-tático individual dos jogadores de futsal das categorias sub-13 e sub15. Rev. Bras. Cien. Mov., Taguatinga, v. 22, n. 2, p. 96-105, mai/ago. 2014.

17. SAAD, M. et al. Estrutura das sessões de treinamento técnico-tático de equipes de futsal sub-13 e sub-15 ao longo da temporada esportiva. Revista Brasileira de Futsal e Futebol, São Paulo, v. 7, n. 25, p. 360-365, mai/ago. 2015. 
A produção científica sobre treinadores de futsal no Brasil

Cesar Vieira Marques Filho - Gustavo Sanchez Folhas • Larissa Rafaela Galatti • Wilton Carlos de Santana $\cdot$ Paulo Cesar Montagner

18.SANTANA, W.; Entrevista Realizada com Ricardo Lucena Técnico campeão da Liga Nacional de Futsal. Conexões, Campinas, v. 10, n. 1, p. 170-175, jan/abr. 2012.

19.SILVA, G. H. G.; GADELHA, L. F. B.; ROLIM, R. M.; Percepção de atletas escolares de futsal sobre o estilo de liderança do treinador. Coleção Pesquisa em Educação Física,Várzea Paulista, v.15, n. 4, p. 139-148, out/dez. 2016.

20.TEIXEIRA, A.; SILVA, S. Parâmetros do processo de ensino-aprendizagem no contexto de treinamneto do futsal. Revista Brasileira de Futsal e Futebol, Edição Especial: Pedagogia do Esporte, São Paulo, v. 7, n. 27, p. 531-539, jan. dez. 2015.

21.TOLVES, B.; DELEVATI, M.; SAWITZKI, R.; Métodos parcial, global e de jogos condicionados no ensino do futsal. Revista Mackenzie de Educação Física e Esporte, São Paulo, v. 13, n. 2, p. 80-92, jul/dez. 2014.

22.VARGAS, L.; CAPUTO, E.; SILVA, M.; Caracterização do perfil dos treinadores de futsal feminino de equipes que disputam os jogos abertos de pelotas. Revista Brasileira de Futsal e Futebol, São Paulo. v. 9, n. 33, p.151-159, maio/ ago. 2017.

23.VARGAS, T. et al.. A experiência do Sport Education nas aulas de educação física: utilizando o modelo de ensino em uma unidade didática de futsal. Movimento, Porto Alegre, v. 24, n. 3, p. 735-748, jul/set. 2018.

24.VOSER, R. et al. A motivação para prática do futsal: um estudo com atletas na faixa etária entre 13 a 18 anos. Revista Brasileira de Futsal e Futebol, São Paulo, v. 8, n. 28, p.3945, jan/abr. 2016.

25. SANTANA, W. C.; FRANÇA, V. S.; REIS, H. H. B.; Perfil do processo de iniciação ao futsal de jogadores juvenis Paranaenses. Motriz, Rio Claro, v. 13, n. 3, p. 181-187, jul/set. 2007. 
A produção científica sobre treinadores de futsal no Brasil

Cesar Vieira Marques Filho • Gustavo Sanchez Folhas • Larissa Rafaela Galatti • Wilton Carlos de

Santana $\cdot$ Paulo Cesar Montagner

26.ZAMBON, S.; BOLSONARO, J. A iniciação do futsal nas escolas de ensino fundamental II em Monte Alto-SP. Revista Brasileira de Futsal e Futebol, Edição Especial: Pedagogia do Esporte, São Paulo, v. 8. n. 31, p.326-333, jan./dez. 2016.

\section{Financiamento}

O presente trabalho foi realizado com apoio da Coordenação de Aperfeiçoamento de Pessoal de Nível Superior - Brasil (CAPES).

\section{Publisher}

Universidade Federal de Goiás. Faculdade de Educação Física e Dança. Publicação no Portal de Periódicos UFG. As ideias expressadas neste artigo são de responsabilidade de seus autores, não representando, necessariamente, a opinião dos editores ou da universidade. 